\title{
An improved itinerary recording protocol for securing distributed architectures based on mobile agents
}

\author{
Guillaume Allée ${ }^{\mathrm{a}}$, Samuel Pierre ${ }^{\mathrm{a}, *}$, Roch H. Glitho ${ }^{\mathrm{b}}$ and Abdelmorhit El Rhazi ${ }^{\mathrm{a}}$ \\ ${ }^{\mathrm{a}}$ Mobile Computing and Networking Research Laboratory (Larim), Canada \\ ${ }^{\mathrm{b}}$ Ericsson Research Canada, Canada
}

\begin{abstract}
This paper proposes an improved itinerary recording protocol for securing distributed architectures based on mobile agents. The behavior of each of the cooperating agents is described, as well as the decision process establishing the identities of offenders when an attack is detected. Our protocol is tested on a set of potential attacks and the results confirm our assumption regarding offender designations and moments of detection. More precisely, the performance evaluation shows that our protocol detects the attack where there is collaboration between a platform on the cooperating agents' itinerary and another on the mobile agent's itinerary. As a result, this protocol constitutes a suitable option for electronic commerce applications where security concerns prevail over cost factors.
\end{abstract}

Keywords: Mobile agent, itinerary recording, distributed architecture, security protocol, electronic commerce, cooperating agents

\section{Introduction}

Wide-area networks allow the design of interesting shared applications that permit constant access to resources and information available on the Internet. However, programming these applications remains challenging: the main problem is due to the fact that the static features of the client-server architecture are not well adapted to the dynamic aspects and diversity of the systems that are available on the Internet. An alternative could be to replace the interactions between both entities by the mobility of an agent that migrates to hosts providing interesting data. Since the agents execute within the environment provided by the remote host, security concerns are paramount, especially for applications like electronic commerce. The problem we are addressing is that of verifying and recording the identities of the host platforms which have executed the agent. This problem is hard to solve with only one agent as malicious hosts can alter the agent's data. One possible solution to detect these attacks would be to use co-operating agents in a protocol to record and verify the itineraries.

Greenberg et al. [11] define the word agent as an autonomous application which has one or several purposes or a set of capabilities and could, if need be, collaborate or communicate with other agents

${ }^{*}$ Corresponding author: Samuel Pierre, Department of Computer Engineering, Ecole Polytechnique de Montréal, C.P. 6079, Station Centre-ville, Montréal, Québec, Canada H3C 3A7. Tel.: +514 3404711 ext. 4685; Fax: +514 340 3240; E-mail: samuel.pierre@polymtl.ca. 
or users. An agent is considered mobile when it can be transported from one machine to another on a network [12]. However, a mobile agent cannot transport itself: it is the executing platform that sends it to another platform through the network. The set of platforms where the agent is executed is called the agent itinerary.

A platform is concurrently a client and a server that allows the execution of an agent. The platform receives and sends the agent. It must be installed on all hosts where the agent stops. Platform protection] techniques are required to shield the execution environment and platform resources against several types of attacks from a mobile agent. On the other hand, agent protection techniques must be used to protect the agent's code and data from interference by malicious hosts $[6,7,9,14,17]$.

Platforms are susceptible to a variety of possible agent attacks. A hostile agent can attempt to access resources without the platform authorization, it may consume too many resources (e.g. CPU or disk space) or disguise itself as another agent. Researchers have developed several techniques in order to protect the platform. Most of these are based on conventional protection approaches but there are some techniques that use new concepts, such as codes with proofs, which consists of asserting certain features of the agent's code (such as memory protection) and supplying a proof of these features. When an agent arrives on a new platform, the latter verifies the compatibility of the proof and the agent's code $[2,3,8]$.

Platform attacks against the agent are even more critical since the platform has access to an agent's components and can modify them in the absence of attack detection or prevention techniques. An attack [13] may take various shapes: a change that modifies the behavior of an agent or alters the stored results obtained from another platform, as well as the denial of services when a platform requests a task from an agent, namely when a platform refuses to send an agent to another platform. A simple yet effective attack on a mobile agent aims at preventing the agent from migrating to competitors' servers. This particularly affects mobile agents with loose itineraries in comparison to agents whose itineraries are defined a priori [15]. The problem consists of verifying and recording the identities of the platforms, which have executed the agent in order to secure the mobile agent's route. It is crucial to detect or protect the mobile agent against this type of attack in the field of e-commerce where the agent wants to visit all of the competitors' servers.

Roth [16] identifies flaws in some cryptographic protocols that are targeted at protecting free-roaming mobile agents, e.g. mobile agents that are free to choose their next hop dynamically based on data they acquire during their execution. He found that some protocols succumbed to interleaving attacks which are impersonation or other deception involving selective combination of information from one or more previous or simultaneously ongoing protocol execution. He presented in [17] an approach which is robust against interleaving attacks. He also introduced algorithms and data structures meant to protect free-roaming mobile agents against attacks on data integrity and confidentiality.

In [4], Westhoff et al. propose a method to protect agent routes against attacks performed by a single platform as well as attacks conducted by collusions of cooperating malicious platforms. They define the route protection as the guarantee that "none of the visited stations can manipulate the route in a malicious ways, nor can they get an overview of the other sites the agent's owner is contacting". They suppose that the agent route is initially provided by the platform of origin and that it can be extended by a visited site. The information related to a mobile agent's route is hidden in such a way that only the mobile agent's platform of origin knows all the platforms to be visited. The platform of origin can be sure that all chosen platforms have been visited. Actually, this method differs from our method as we consider the agent route to be totally dynamic (i.e., the route is not fixed by the platform of origin).

Schneider [5] combines the replication and the voting concepts to ensure the proper functioning of the agent. The idea is that instead of having a computation performed solely by the mobile agent, multiple 
copies are used to perform the same computation and the result is obtained by a result consensus from all of the platforms. If a malicious platform attacked the mobile agent, the existence of several clones would ensure the correct end result. The main advantage of this method is that it addresses the system failure. Its drawbacks is that additional resources are needed for agent replications. The method proposed in this paper also uses the concepts of replication to ensure security i.e. a cooperating agent executes partially the same code as the one executed by the mobile agent.

This paper proposes a security mechanism inspired by Roth [15] to protect mobile agents' itineraries against a set of potential attacks from offender platforms in an electronic commerce context. Section 2 summarizes Roth's protocol. Section 3 proposes a new itinerary recording protocol (based on Roth's protocol) and assesses the effectiveness of this new protocol through the types of attacks it detects. Section 4 presents implementation details and results.

\section{Summary and background of Roth's protocol}

Itinerary recording is carried out according to a protocol whose purpose is to record and verify the itinerary of a mobile agent with a cooperating agent, in an autonomous fashion, without permanently connecting a trusted platform to the network. The two agents' owner platform connects to the network to send the agents to their first platforms, and then it disconnects itself until both agents return. As the itinerary is not pre-established, the mobile agent moves progressively while accomplishing its tasks and it chooses its next destinations during its execution on different platforms. The purpose of Roth's protocol is to record and verify the mobile agent's itinerary.

\subsection{Definitions and main assumptions}

Let $H$ be the set of available platforms on a network. $R$ is a subset of $H \times H$ such as $\left(h_{i}, h_{j}\right)$ $\in R \Leftrightarrow h_{i}$ and $h_{j}$ collaborate in order to attack the agent. $H_{a}$ and $H_{b}$ are non-void subsets of $H$ with $\left(H_{a} \times H_{b}\right) \cap R=\varnothing$. These two subsets are considered non-collaborating sets of platforms. Two agents, $a$ and $b$, are considered cooperating agents, when the itinerary of agent $a$ contains only $H_{a}$ platforms and agent $b$ 's itinerary has only $H_{b}$ platforms. $h_{a}$ and $h_{b}$ are current execution environments for agents $a$ and $b$. Basically, agent $a$ could be attacked by platform $h_{a}$ that executes it, but $h_{a}$ could not directly attack cooperating agent $b$. Moreover, platform $h_{b}$ will not collaborate with $h_{a}$ for this purpose. However, it is possible that two platforms on agent a's itinerary collaborate to attack it.

Roth [14] justifies this choice by stating that it is as unrealistic to state that "all platforms are hostile and ready to collaborate with each other to attack the agent" as to claim that "all platforms could be securely used". According to Roth, it is more realistic to state that:

- At a given moment in the life of an agent, a certain percentage of platforms could be considered dangerous;

- All dangerous platforms are not necessarily ready to collaborate with other platforms to attack an agent.

Roth [14] adds the following three assumptions to implement the concept of cooperating agents:

- The transport of agents from one platform to another is carried out through an authenticated channel (Assumption 1);

- The platforms supply authenticated communication channels to cooperating agents (Assumption 2);

- The agent has authenticated access to the identity of the remote platform with which it communicates, the platform on which it is executed, and to the previous platform on which it was executed (Assumption 3). 
Definition: Let $h_{i} \in \mathrm{H}_{\mathrm{a}}$ be the platforms visited by agent $a$ and let $i d\left(h_{i}\right)$ be the identity of platform $h_{i}$. Let prev $_{i}$ be the identity of the last platform where agent $a$ has been executed. Finally let next $t_{i}$ be the identity of the next platform where the agent would like to go after platform $h_{i}$. The agent starts from platform $h_{0}$. Thus, if the agent moves a total of $n$ times, we must obtain $h_{0}=h_{n}$, since agent $a$ returns to the platform of origin.

Initialization: Let $h_{0}$ be the platform of origin of agents $a$ and $b . h_{0}$ must be a trustworthy platform for agents $a$ and $b$. Each agent is sent to its first platform (next $\left.t_{0}\right)$ through an authenticated communication channel (Assumption 1).

Step $i, i \in\{1, \ldots, n\}$ :

Agent $a$ sends a message to agent $b$ containing next ${ }_{i}$ and prev $_{i}$ through an authenticated channel (Assumption 2). Thus, agent $b$ learns $i d\left(h_{i}\right)$ (Assumption 3) and verifies that

$$
i d\left(h_{i}\right)=\text { next }_{i-1} \text { and } \text { prev }_{i}=i d\left(h_{i-1}\right)
$$

If this is the case, b records next $t_{i}$ in agent a's itinerary.

Fig. 1. Itinerary recording protocol.

\subsection{Roth's protocol}

Let $a$ and $b$ be two cooperating agents, $H_{a}$ and $H_{b}$ two non-collaborating platform subsets of $H$. Each agent must return to its platform of origin at the end of its task. Agent $b$ records and verifies the itinerary of its cooperating agent $a$, according to the protocol described in Fig. 1.

The need for a cooperating agent is mainly justified by the fact that a malicious platform might modify the data held by an agent executing on that platform. Indeed, it could be argued that, if one supposes that the third assumption is valid, it is sufficient that the agent keeps the authenticated identity of each platform where it was executed. It could be supposed that it makes the same verifications as agent $b$ in Roth's protocol. However, this solution is not secure. The following is a contrary example with an undetected attack. Host $h_{i}$ attacks the agent by sending it to host $h_{f}$, although the agent is supposed to go to platform $h_{i+1}$. The identity of host $h_{f}$ is added to the itinerary record, then host $h_{f}$ replaces the agent on its route by sending it to $h_{i+1}$, which is supposed to be honest. On $h_{i+1}$, the agent verifies the identity of the platform that sent it, which appears correct, since $h_{f}$ did not modify the itinerary record. On step $j>i+1$, host $h_{j}$, which is allied with attacking platforms $h_{i}$ and $h_{f}$, modifies the itinerary record to remove the record of execution on host $h_{f}$. When the agent returns to its owner's platform, its itinerary record appears correct although there are no records of the execution on host $h_{f}$.

\section{Proposed new itinerary recording protocol}

It should be noted that Roth's protocol does not specify the manner by which agent $b$ moves. In our approach, we propose to move agent $b$ every time agent $a$ moves, i.e., when agent $a$ contacts $b$ to announce the identities of its next and previous execution platforms. Once this information is obtained, 
agent $b$ moves to another platform and waits to communicate with agent $a$. We will specify the required assumptions for our protocol, describe the possible attacks, and indicate the manner in which they will be detected by the protocol. Finally, we will show the originality of our protocol in relation to Roth's.

\subsection{Assumptions}

As stated above, agent $b$ will move. There are two possibilities for agent $b$ 's itinerary: it is either pre-determined or not. We propose to fix the itinerary of agent $b$ at the beginning of the protocol. The advantage of this choice is that, with a fixed itinerary, we simplify the protocol and increase the likelihood of detecting the attack.

We suppose that agent $a$ has a chronological list of platforms to be visited by agent $b$. We call this list $p f, p f[i]$ indicating the platforms visited by agent $b$, later called $h b_{i}$. Since a movement from agent $a$ triggers the movement of agent $b$, the number of platforms visited by agent $b$ must be identical to the number of platforms visited by agent $a$. However, the number of hosts visited by agent $a$ cannot be predicted. To solve this problem, the number of platforms visited by agent $a$ will be limited. This type of estimate is possible when "looking for the best price for a product" type of applications, where we know that the agent will visit a number of vendors, but we know neither the platforms (because a vendor has several platforms) nor the order in which the visits will take place. Therefore, it is impossible to establish an itinerary before the departure from the owner's platform. Agent $b$ 's itinerary consulted by agent $a$ must be protected from modifications. This itinerary could, for example, be part of the agent's data and thus, with the agent's code, could be protected from modifications. This is possible with the owner's signature guaranteeing the integrity of the agent and publishing its identity for the benefit of the platforms where the agents will eventually be executed.

We suppose that the set of platforms visited by agent $a$ and the set of platforms visited by agent $b$ are disjoint. The order in which agent $b$ visited the platforms is irrelevant. It is nevertheless important to minimize the probability of an attack from a platform on agent $b$ 's itinerary with a collaboration from $a$ platform on agent $a$ 's itinerary. However, it is difficult to quantify this risk, since $a$ 's itinerary is not pre-determined. This is why agent $b$ 's itinerary should be chosen randomly, from a list of platforms that do not offer the services sought by agent $a$.

Figure 2 illustrates these assumptions using a collaboration example considered impossible in Roth's protocol, which becomes possible in the protocol we suggest. We use the same assumptions as Roth's (cf. Section 2.1). This figure shows the assumptions that we put forth concerning the platform collaboration to perform an attack. In our assumption, the platforms in $a$ 's itinerary and $b$ 's itinerary can collude to attack mobile agent $a$. It is important to note that Roth' assumption stipulates that the platforms in $a$ 's itinerary and $b$ 's itinerary cannot collude to attack mobile agent $a$. For example, on the left of the figure, the platforms $h a_{i}$ and $h b_{i+1}$ can collude to attack agent $a$.

The impossible collaboration between platforms in the itinerary of agent $a$ and platforms in the itinerary of agent $b$ is simply an assumption put forth by Roth. Our protocol treats the case where this collaboration is not supposed. Consequently, our protocol supposes a reduced number of assumptions compared to Roth's protocol and treats more realistic cases.

\subsection{Itinerary recording protocol}

\section{Definition}

Let $h a_{i}$ be the platform visited by agent $a, i d\left(h a_{i}\right)$ the identity of this platform, $h b_{i}$ the platform visited by agent $b$ and $i d\left(h b_{i}\right)$ the identity of this platform. Let prevai be the identity of the last platform 


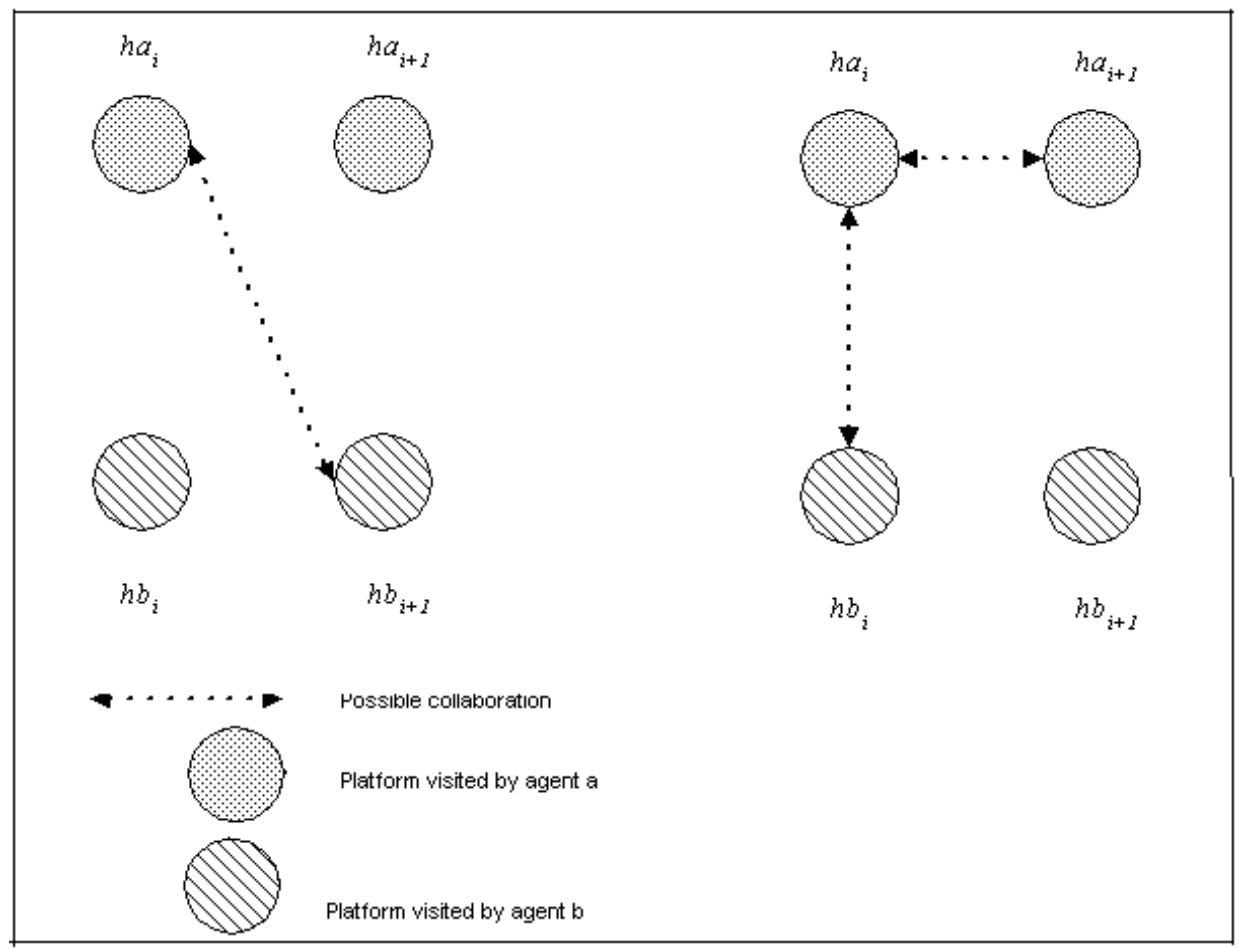

Fig. 2. Possible collaborations.

where agent $a$ was executed. According to Assumption 3, the agent has access to this identity in an authenticated manner. Finally, let nextai be the identity of the next platform that agent $a$ would like to visit after visiting platform $h a_{i}$. The agent begins its itinerary on platform $h a_{0}$. Thus, if agent $a$ moves a total of $n$ times, we obtain $h a_{0}=h a_{n}$ as the assumption stating that the agent finally returns to the platform of origin.

\section{Initialization}

Let $h_{0}$ be the platform of origin of both agents $a$ and $b . h_{0}$ must be a trusted platform for both agents $a$ and $b$. For agent $a$, next $a_{0}$ has the value of the first platform where the agent will be executed. Each agent is sent to its respective destination.

Step $i, i \in\{1, \ldots, n-1\}$

Agent $b$ waits for communication from agent $a$. If agent $a$ does not communicate after a certain period of time, agent $b$ would consider that agent $a$ was kidnapped, and it would return to its owner's platform. The offender cannot be identified with certainty: it could be either that platform $h a_{i-1}$ did not actually send the agent, or that platform $h a_{i}$ hindered communication.

Agent $a$ attempts to communicate with agent $b$ on platform $h b_{i}$.

If agent $b$ is not on this platform, it is due to the fact that the previous platform $h b_{i-1}$ did not send it and that platform $h b_{i}$ did not execute it, or it did not grant network access to agent $b$ in order to receive communication from agent $a$. Therefore, we cannot record nor verify agent $a$ 's itinerary. In this case, agent $a$ returns to its platform of origin. 


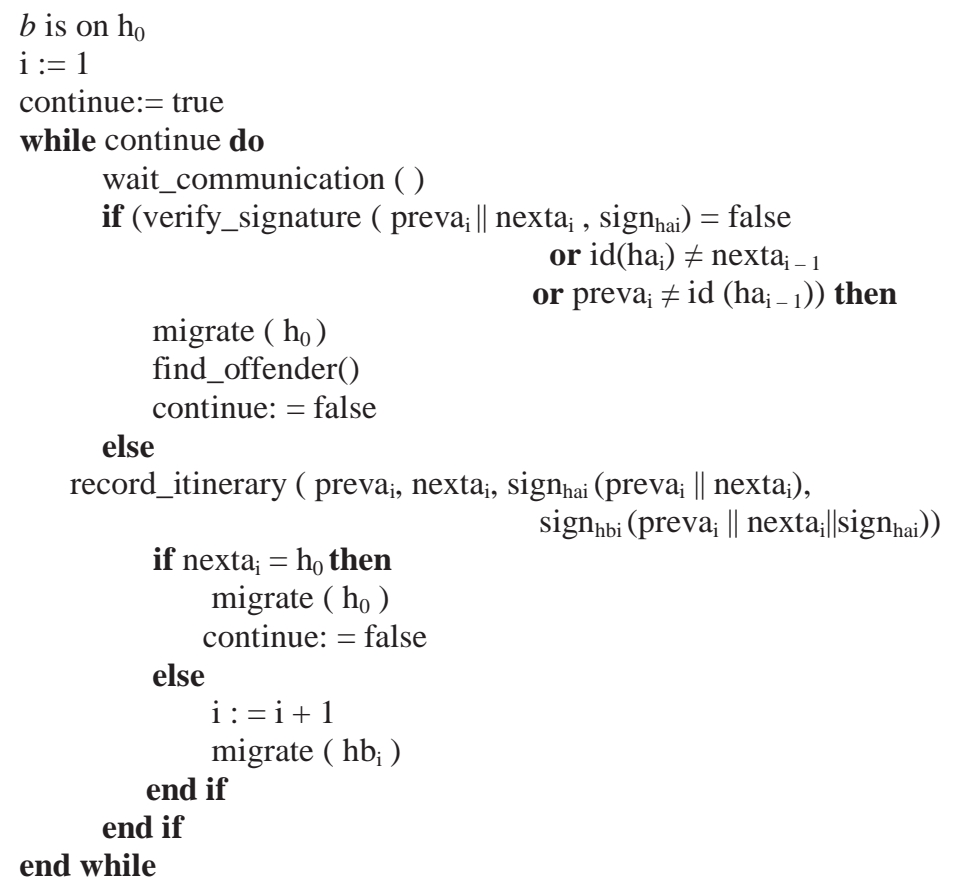

Fig. 3. Algorithm for agent $b$.

Otherwise, agent $a$ sends agent $b$ the identities of next $a_{i}$ and $p r e v a_{i}$, signed by platform $h a_{i}$ and by the authenticated channel, $b$ learns $i d\left(h a_{i}\right)$. It verifies the signature of $h a_{i}$ and the following equalities:

$$
i d\left(h a_{i}\right)=n e x t a_{i-1} \text { and } \operatorname{preva}_{\mathrm{i}}=i d\left(h a_{i-1}\right)
$$

If these equalities are confirmed, agent $b$ records next $a_{i}$, preva $a_{i}, h a_{i}$ 's signature, then requires a signature from $h b_{i}$. We use $\operatorname{sign}_{Y}(X)$ to designate the result of the cryptographic signature of platform $Y$ on object $X$ and we use $\|$ to designate concatenation. Here is a typical entry from the itinerary record.

$$
\operatorname{preva}_{i} \quad \operatorname{nexta}_{i} \quad \operatorname{sign}_{h a i}\left(\operatorname{nexta}_{i} \| \operatorname{preva}_{i}\right) \quad \operatorname{sign}_{h b i}\left(\operatorname{preva}_{i}\left\|\operatorname{nexta}_{i}\right\| \operatorname{sign}_{h a i}\right)
$$

Non-confirmed identities indicate that an attack has occurred. In this case, agent $b$ returns to its owner's platform.

Agent $a$ performs its task on the platform before migrating to its subsequent destination. Figures 3 and 4 describe the algorithms for agents $a$ and $b$ respectively.

\section{Step $n$}

When the agents return to their owner's platform without detecting an attack, the owner verifies whether the itinerary has been modified. For each entry $i \in\{1, \ldots, n\}$, it verifies whether the signature $\operatorname{sign}_{\text {hai }}\left(\right.$ preva $_{i} \|$ nexta $\left.a_{i}\right)$ is valid and that $h a_{i}=$ nexta $_{i-1}=$ preva $_{i+1}$. It repeats the operation for $\operatorname{sign}_{h b i}\left(\operatorname{preva}_{i} \|\right.$ nexta $\left._{i} \| \operatorname{sign}_{h a i}\right)$. An invalid entry indicates the occurrence of an attack on the agent's itinerary and the results are considered invalid. The offender was not identified.

Once the integrity of the itinerary record has been confirmed, the agent's owner verifies whether agent $a$ 's itinerary was executed on the designated platforms. A negative outcome indicates an attack has occurred and messages exchanged between $a$ and $b$ have been modified. 


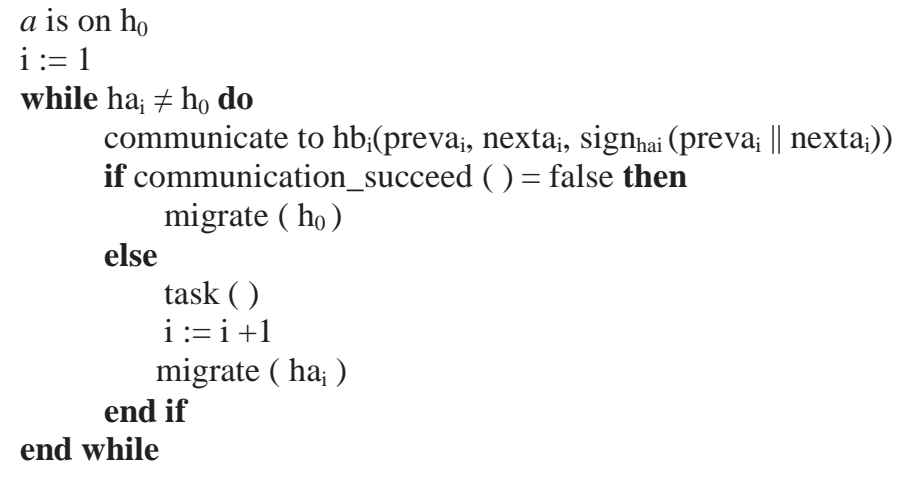

Fig. 4. Algorithm for agent $a$.

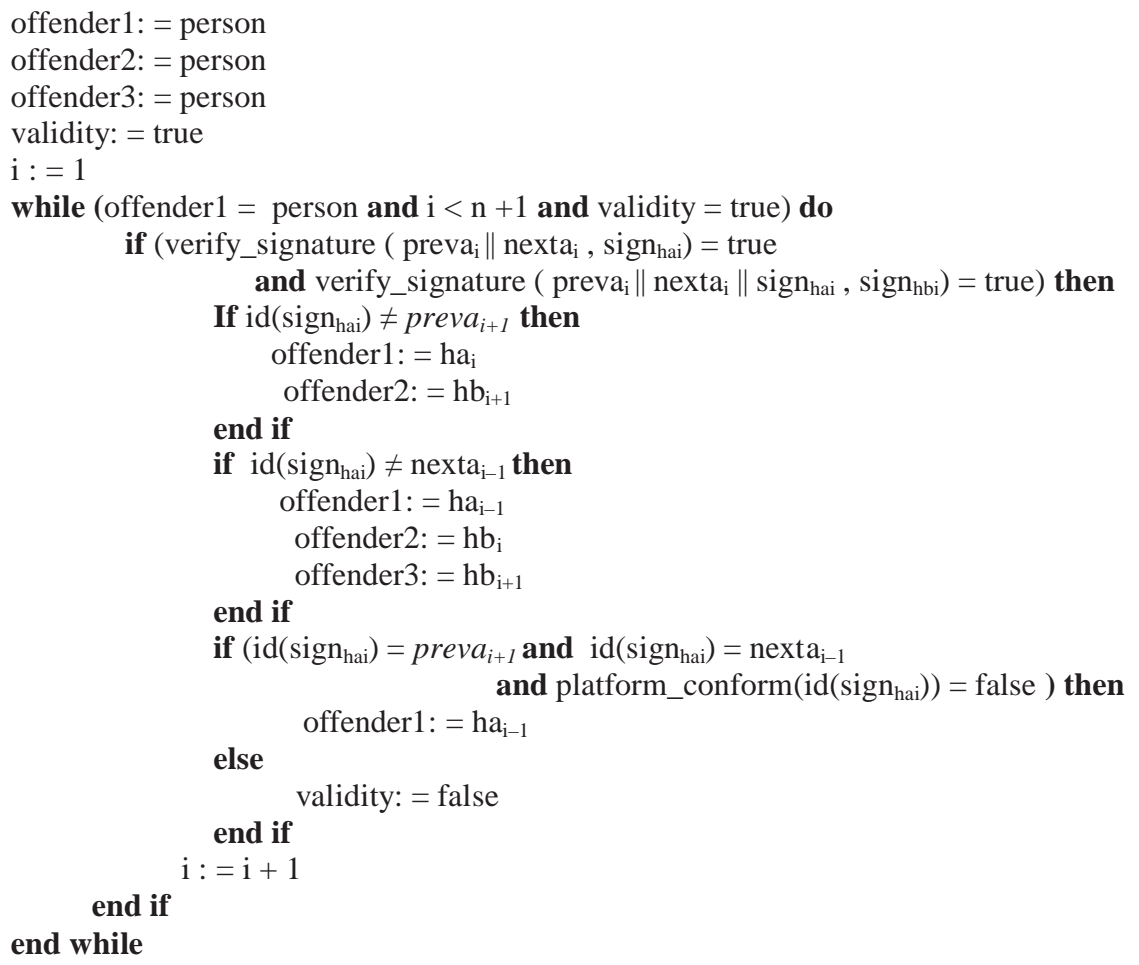

Fig. 5. Algorithm Step $n$.

Figure 6 describes the function find of fender() executed by agent $b$ when it detects an attack before step $n$, while Fig. 5 presents the algorithm executed on step $n$ in the absence of attack detection in the previous steps. When identifying the offender, the algorithm does not consider the man-in-the-middle attack as the protocol supposes that the communication channels between agents $a$ and $b$ are authenticated and secured (e.g., using SSL [3]). 


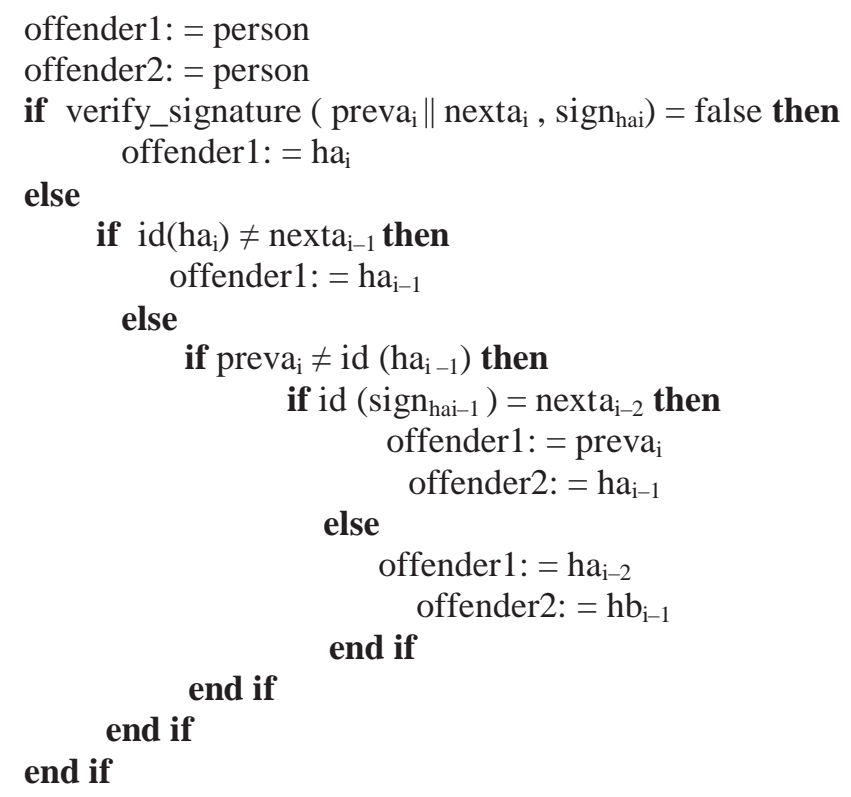

Fig. 6. Function find offender().

\subsection{Protocol security}

The protocol we propose functions similarly to Roth's protocol [15], in that it detects the same attacks when we uphold Roth's assumptions, i.e., if we suppose there is no collaboration. However, with the weaker assumptions that we stated, we obtain identical results at the level of detected attacks. The possibilities for collaboration between two hosts $h a_{i}$ and $h b_{j}$ fall into one of the four subsets: $i<j-1, i=j-1, i=j$, and $i>j$. We will also consider the case of collaboration among the three hosts $h a_{i}, h b_{i+1}$ and $h b_{i+2}$. The other attacks with this type of collaboration can be modeled as two different attacks with collaboration between $a$ 's itinerary platform and agent $b$ 's itinerary platform.

\subsubsection{Attack with Collaboration between $h a_{i}$ and $h b_{j}$, with $i<j-1$}

In this scenario, the platforms $h a_{i}$ and $h b_{j}(i<j-1)$ collude in order to attack the mobile agent by modifying its itinerary. Let's suppose that the mobile agent decides to migrate to platform $h$ ' (refer to Fig. 7) while running on platform $h a_{i}$. Platform $h a_{i}$ attacks the mobile agent $a$ by sending it to platform $h a_{i+1}$ and modifies the message sent by mobile agent $a$ to the cooperating agent $b$ (i.e. replaces the identity of platform $h$ ' by the identity of platform $h a_{i+1}$ in the variable nexta $a_{i}$. With this modification, co-operating agent $b$ cannot detect the attack. However, it will be detected by the platform of origin upon the agents' return to it because the platform of origin will detect that agent $a$ is executed on platform $h a_{i+1}$ instead of $h$ '. In order to prevent the platform of origin from detecting this attack, platform $h a_{i}$ will ask platform $h b_{j}$ to modify the entry corresponding to platform $h a_{i+1}$ in agent $a$ 's itinerary.

Replacing an entry in the itinerary record is impossible, since this latter resists to the modifications. An entry is simultaneously signed by platform $h a_{i}$ and $h b_{i}$. If platform $h b_{j}$ attempts to modify an entry with $j>i$, it could not create a valid entry at the signature level as it cannot imitate the signature of platform $h b_{i}$. Therefore, if it modifies nextai; the signature will be invalid and the attack will be detected when signatures are checked. However, the platform of origin cannot find the offender. If $h b_{i}$ collaborates with 


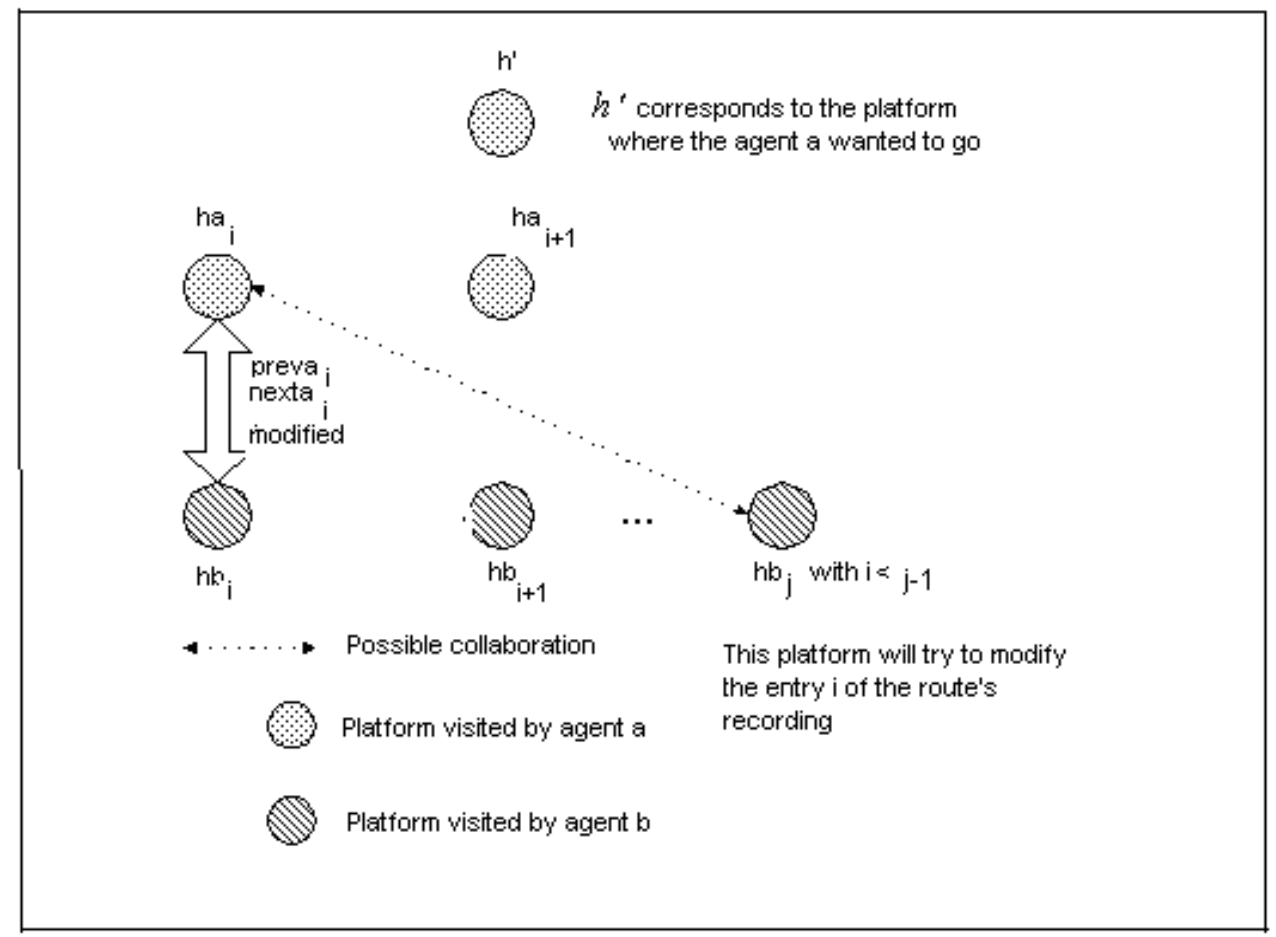

Fig. 7. Attack with collaboration between $h a_{i}$ and $h b_{j}(i<j-1)$.

$h b_{j}$ to counterfeit its signature, the attack will, nevertheless, be detected upon the agent's return, since the correspondence between nexta $a_{i}$ and the identity of the platform that signed entry $i+1$ is invalid.

If platform $h b_{j}(j>i)$ could create its own agents and collect suitable records from the correct itinerary of agent $a$, it could replace the entry $i$. Consequently, the protocol cannot detect this scenario of attack due to the fact that the information route recorded by agent $b$ are not linked to a particular agent instance.

\subsubsection{Attack with collaboration between $h a_{i}$ and $h b_{i+1}$}

If $h a_{i}$ sends agent $a$ to platform $h a_{i+1}$ with $i d\left(h a_{i+1}\right) \neq n e x t a_{i}$, platform $h b_{i+1}$ will receive $i d\left(h a_{i+1}\right)$, preva $_{i+1}$ and nexta $a_{i+1}$ information, then verify the equality of $i d\left(h a_{i+1}\right)=$ nexta $a_{i}$. However, unless the authentication key was stolen, this equality cannot be confirmed. Therefore, agent $b$ (on platform $h b_{i+1}$ ) detects a problem on the itinerary. However, it is possible that this platform collaborates with hai to have agent $b$ believe that this equality is true. Therefore, $h a_{i}$ collaborates with $h b_{i+1}$. Agent $b$ carries out equality verifications at step $i+1$, although the error remains undetected due to manipulation from the platform on which it is executed, $h b_{i+1}$. Agent $b$ finds that the two equalities are verified. Once on platform $h b_{i+2}$, the verification of the equality preva $_{i+2}=i d\left(h a_{i+1}\right)$ indicates an error since platform $h a_{i}$ did not send the agent to nexta $a_{i+1}$. Unmasking the offender would be problematic since this equality is not verified. Moreover, this corresponds to the case where host $h a_{i+1}$ sends the agent to a wrong platform, which would attempt to put it on its track. Figure 8 illustrates this attack scenario. Indeed, if $h a_{i+1}$ sends agent $a$ to a different platform than nexta $a_{i+1}$, and that this platform sends agent $a$ towards identity platform nexta $a_{i+1}$ to put the agent on its track, once on platform $h a_{i+2}$, the equality $\operatorname{prev}_{i+2}=i d\left(h a_{i+1}\right)$ will not be verified, because the latter authentically knows the identity of the platform that sent it to agent $a$. 


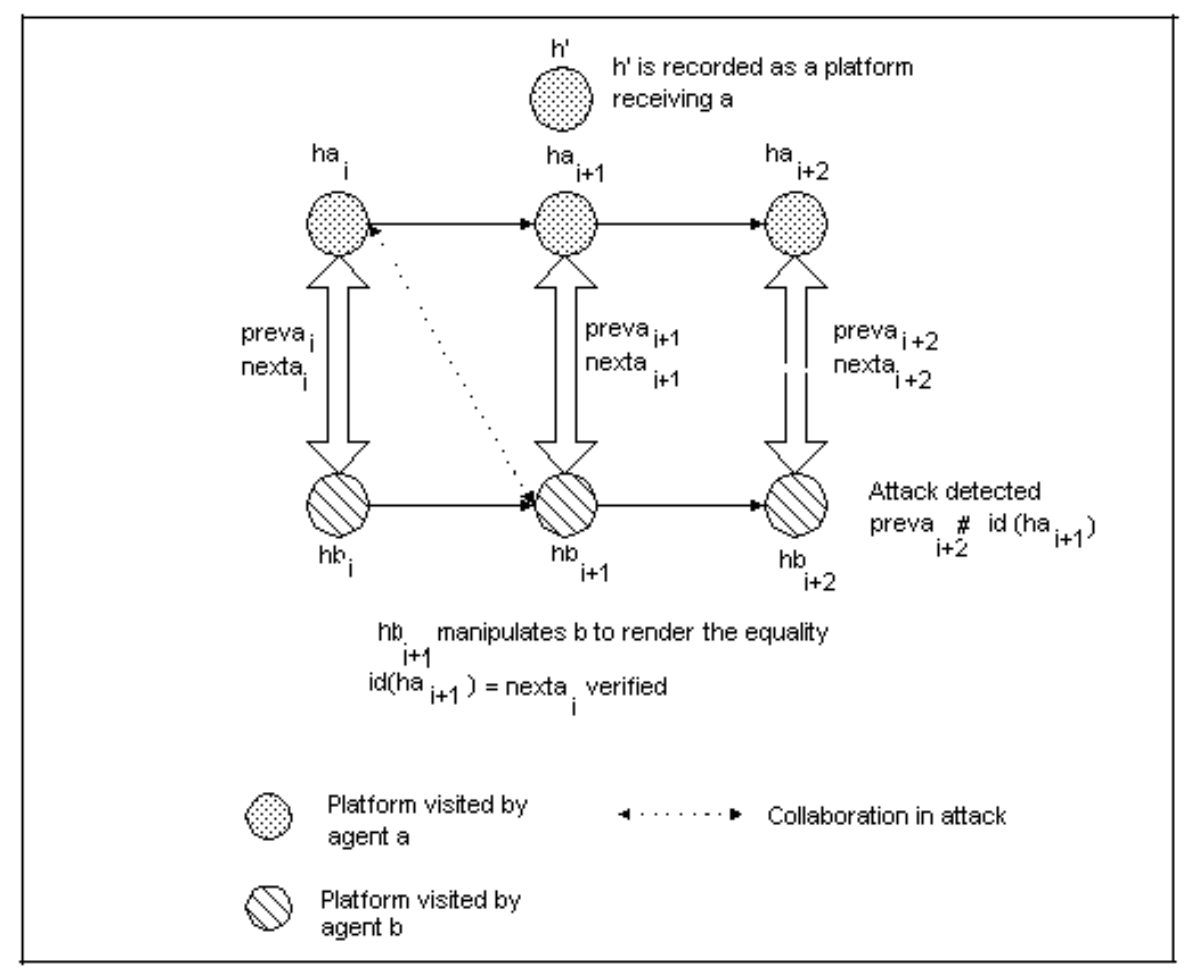

Fig. 8. Attack with collaboration between $h a_{i}$ and $h b_{i+1}$.

Thus, finding a culprit is problematic: we know it is possible that the offender would be $h a_{i}$, this is the case we studied with $h b_{i+1}$ collaboration, or it could be that $h a_{i+1}$ (with an accomplice) tried to put the agent on its track. Agent $b$ verifies nexta $a_{i+1}$ signature by $h a_{i+1}$ and compares this identity with nexta $a_{i}$. This is carried out in the function find of fender (), which is executed when the agent detects the attack. If this is a first attack, the identity of the platform that signed nexta $a_{i+1}$ is different from both next $a_{i}$ and offender $h a_{i}$, with the collaboration of $h b_{i+1}$. In the case of a second attack, the result is an equality between these two entities and the offender is $h a_{i+1}$.

Another attack with collaboration between $h a_{i}$ and $h b_{i+1}$ can occur. Platform $h a_{i}$ sends agent $a$ to $h$, whose identity is different from the host where it would really like to send agent $a$. Then, host $h$ ' would try to put the agent on its track by sending it to $h a_{i+1}$. Once on $h a_{i+1}$, agent $a$ communicates with agent $b$ to give it preva $_{i+1}$ and nexta $a_{i+1}$. Agent $b$ carries out its verifications and would normally deduce that the agent deviated from its trajectory since $\operatorname{preva}_{i+1} \neq i d\left(h a_{i}\right)$. However, $h b_{i+1}$ manipulates it in order to confirm this equality.

Although this attack is not immediately detected, it will be noticed when the agent returns to its platform of origin. On step $n$, the verification of the itinerary record will establish that $i d\left(\operatorname{sign}_{\text {hai }}\right) \neq$ preva $_{i+1}$ and offenders $h a_{i}$ and $h b_{i+1}$ will be unmasked. If $h b_{i+1}$ modifies the record of entry $i+1$, it will be incapable of reproducing $h a_{i+1}$ signature. Thus, $h b_{i+1}$ cannot do so without detection upon the agent's return as the validity of the signatures is verified on step $n$. However, in this case, the identity of the offender is revealed. 


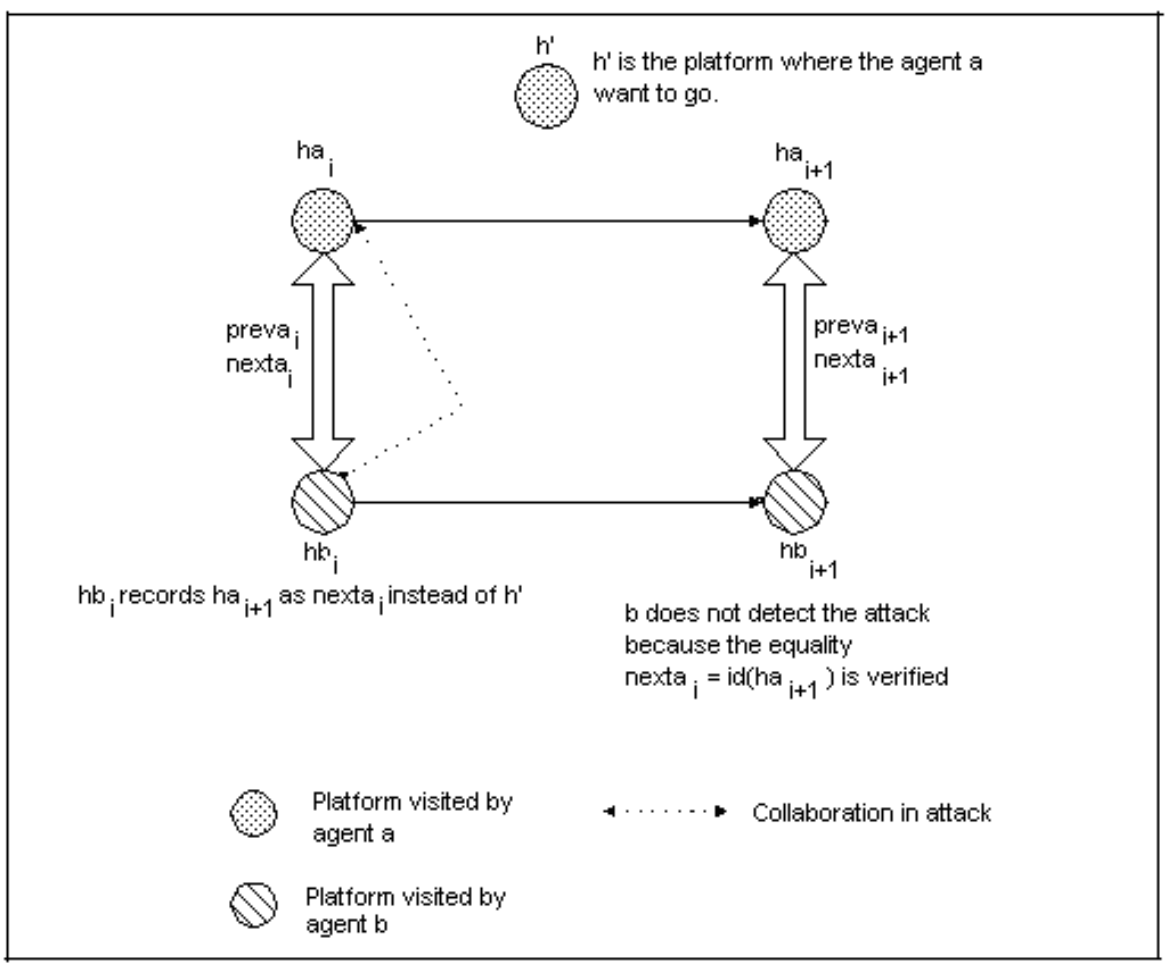

Fig. 9. Attack with collaboration between $h a_{i}$ and $h b_{i}$.

\subsubsection{Attack with collaboration between $h a_{i}$ and $h b_{j}$, for $j=i$ and for $i>j$}

Suppose that $h a_{i}$ collaborates with $h b_{i}$ so that agent $b$ records $h_{f}$ (where the attacking platform $h a_{i}$ would like to send agent a) as the identity of platform $i+1$ visited by agent $a$ instead of $h$ ' (where $a$ would like to go). Then, agent $b$ records a next $a_{i}$ which is false, and platform $h a_{i}$ sends the agent to the platform that was recorded. In this case, when $b$ will be on platform $h b_{i+1}$, (that we suppose to be honest), it will receive the following information from $a$ : nexta $a_{i+1}$, preva $_{i+1}, i d\left(h a_{i+1}\right)$. The verification of equality nexta $a_{i}=i d\left(h a_{i+1}\right)$ is carried out and does not allow immediate detection of the attack since platform $h b_{i}$ recorded where platform $h a_{i}$ sent it $\left(h a_{i+1}\right)$ rather than agent $a$ 's original destination $\left(h^{\prime}\right)$. However, this type of fraud will be detected upon the agent's return to its platform of origin, since agent $b$ kept the complete itinerary of a and the agent's owner verifies this itinerary. Figure 9 shows this scenario of attack. This is not a drawback of our protocce this type of fraud is similar to the case where a malicious platform counterfeits next $a_{i}$, in the communication between $a$ and $b$ to send agent a toward another platform than its destination, without $b$ being able to detect the attack. This attack was called "attack with modification of communication between $a$ and $b$ " in Roth's protocol. The latter also does not allow for an immediate detection of this attack, although it detects the attack on step $n$, by verifying agent $a$ 's itinerary upon agent $b$ 's return. If $h a_{i+1}$ is dishonest, the platform must attempt to replace the agent on its track. This is detected with $h b_{i+1}$ since the equality preva $+i+2=i d\left(h a_{i+1}\right)$ will not be verified. A collaboration between $h a_{i}$ and $h b_{j}$ for $i>j$ prevents an attack. Indeed, agent $b$ is executed on host $h b_{j}$ before agent $a$ migrated to host $h a_{i}$. 


\subsubsection{Attack with collaboration between $h a_{i}, h b_{i+1}$ and $h b_{i+2}$}

The principle of this attack is identical to the attack with collaboration between $h a_{i}$ and $h b_{i+1}: h a_{i}$ sends agent $a$ towards a different platform than its original destination; once on the platform, agent $a$ communicates with agent $b$ to access the identities of the platform, as specified in the protocol. Figure 10 illustrates this scenario of attack. Usually, $b$ would detect the attack, but in this instance, it has been manipulated by platform $h b_{i+1}$ that collaborates with $h a_{i}$. Therefore, it does not detect the attack and migrates towards host $h b_{i+2}$. In the case where $h b_{i+2}$ was honest, we would have immediately detected the attack and found the offender. Here, $h b_{i+2}$ collaborates and manipulates agent $b$ to have it believe that equality preva $a_{i+2}=i d\left(h a_{i+1}\right)$ is verified. In this case, the agents' return to their owner's platform should be expected. The algorithm executed upon the return of the agent to their owner's platform (step $n$ ) compares the identity of the platform on agent $a$ 's itinerary, who signed the entry $i+1$ with nextai identity. next $a_{i}$ is different from the identity of the platform that signed entry $i+1$ of itinerary $\left(h a_{i+1}\right)$. If this attack was previously undetected, it is due to the collaboration between $h a_{i}, h b_{i+1}$ and $h b_{i+2}$. As indicated earlier, if $h b_{i+2}$ is honest, the platform will detect the attack and unmask the offenders.

\subsubsection{Other attacks}

Some other attack scenarios could be imagined. However, they will involve the collaboration of several platforms, and are consequently not addressed in this paper. For example, spoofing agents $a^{\prime}$ and $b^{\prime}$ could be created by $h a_{n-1}$ and $h b_{n-1}$. The aim of these agents would be to revisit the itinerary and correct it. This scenario involves the collaboration of $h a_{n-1}, h b_{n-1}, h a_{1}, h b_{1}$ and $h b_{i}$ (the platform that modifies the agent's itinerary). The protocol cannot detect the modification of agent $a$ 's itinerary. Nevertheless, even if this latter could be corrected, the results obtained inside the visited platform remain invalid in the case where they are signed by the platform.

\section{Implementation and Results}

The platform used to implement our protocol is Grasshopper $2.2 .3,{ }^{1}$ which uses Java as the agent programming language. To carry out the tests, we used 5 machines running Windows 2000. The machines are identified with the name used for the shopping application. The network used is a local network, Ethernet $100 \mathrm{Mbps}$. The mobile agents are implemented using JDK 1.3. ${ }^{2}$ The security package IAIK-JCE 3.01 [10] was used; it offers a set of different cryptographic algorithms implemented in pure Java.

It was deemed important to test our protocol in a relatively realistic environment and we decided to implement an application where the recording and itinerary verification could help secure the application. Our agent shops for a certain number of products on the user's behalf. Provided with a description of the products required by the user, it moves to the platform of many vendors to inquire about their prices. In order to simplify the presentation, the list of products is supposed to be known by the vendors.

The itinerary is dynamic: initially, the user starts the search by entering a certain number of vendor addresses. However, while shopping, if a vendor does not have the listed products, the agent is referred to another vendor with whom trade agreements have been established. These agreements contain financial compensation clauses for the benefit of the vendor who routed the agent to another address.

\footnotetext{
${ }^{1}$ http://www.grasshopper.de/ (July 2003).

${ }^{2}$ http://java.sun.com/j2se/1.3/ (July 2003).
} 


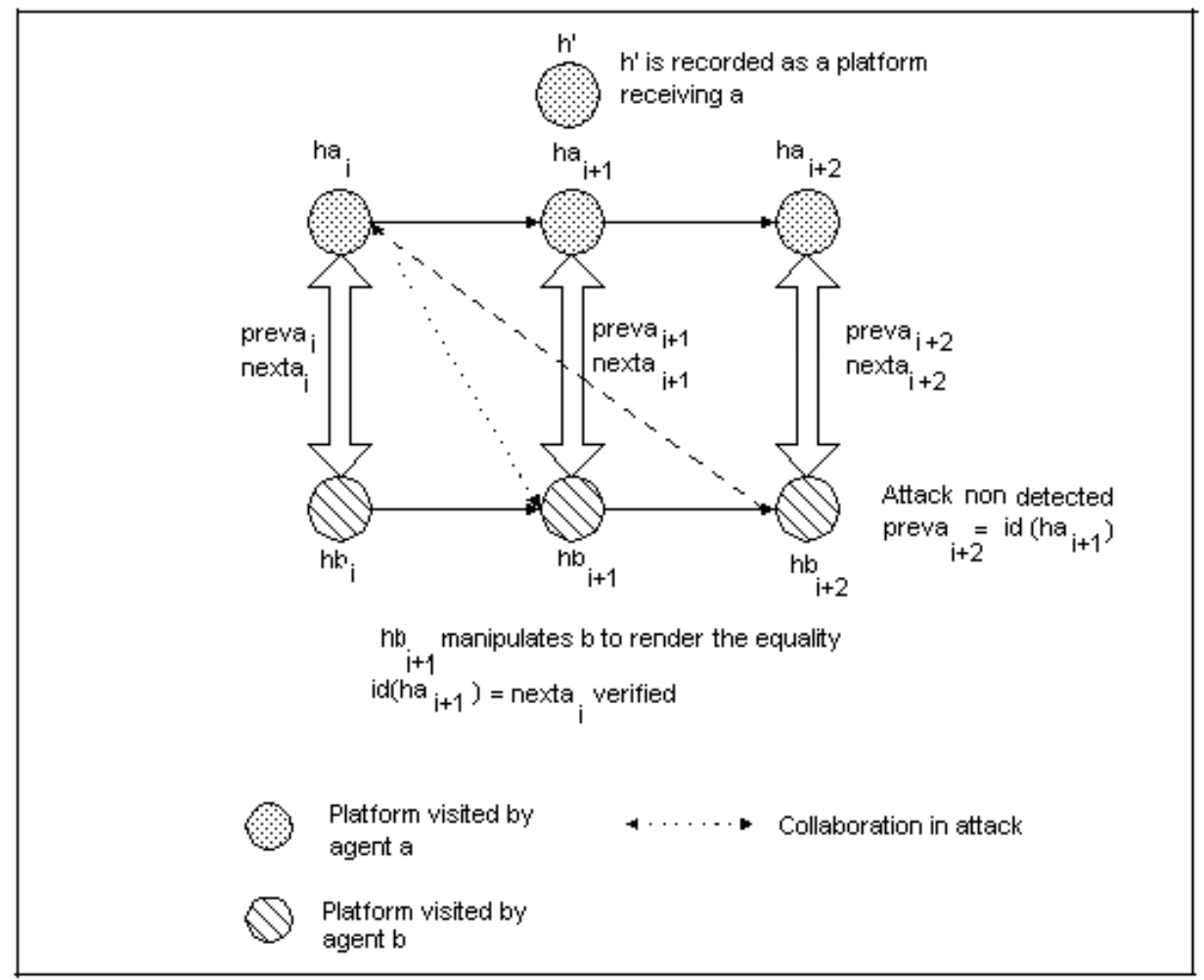

Fig. 10. Attack with collaboration between $h a_{i}, h b_{i+1}$ and $h b_{i+2}$.

The mobile agent records the three best offers for each product sought. It could save the entire set of offers, but if they are too numerous, the result size would become too large. In this case, the agent could lose some of its advantages over the client-server approach. We chose to save the best three results instead of a single one, since factors other than prices may influence the user's choice (the user could choose a slightly more expensive product if it came from a more reputed company for instance). The Grasshopper's transport service allows for the agents' transport via SSL (Secure Sockets Layer).

\subsection{Implementation tests}

First, we tested the agent's authenticated transport communication. For the client, as well as for the server, we tested three different cases:

- the client or the server has a signed certificate issued by the certification authority, which is considered trustworthy by the other entity;

- the client or the server has a signed certificate issued by the certification authority that is not considered trustworthy by the other entity;

- the client or the server does not have a certificate.

The SSL connection is refused when the client's or the server's certificate is not trustworthy. Since the mutual authentication was forced through our SSL connection, this confirms our assumptions.

Then, we tested the authenticated communication between the two agents. The agent transport is carried out only if the agent and server have a certificate signed by a trustworthy certification authority. In the other cases, the SSL connection is refused and the message is not sent from agent $a$ to agent $b$. 


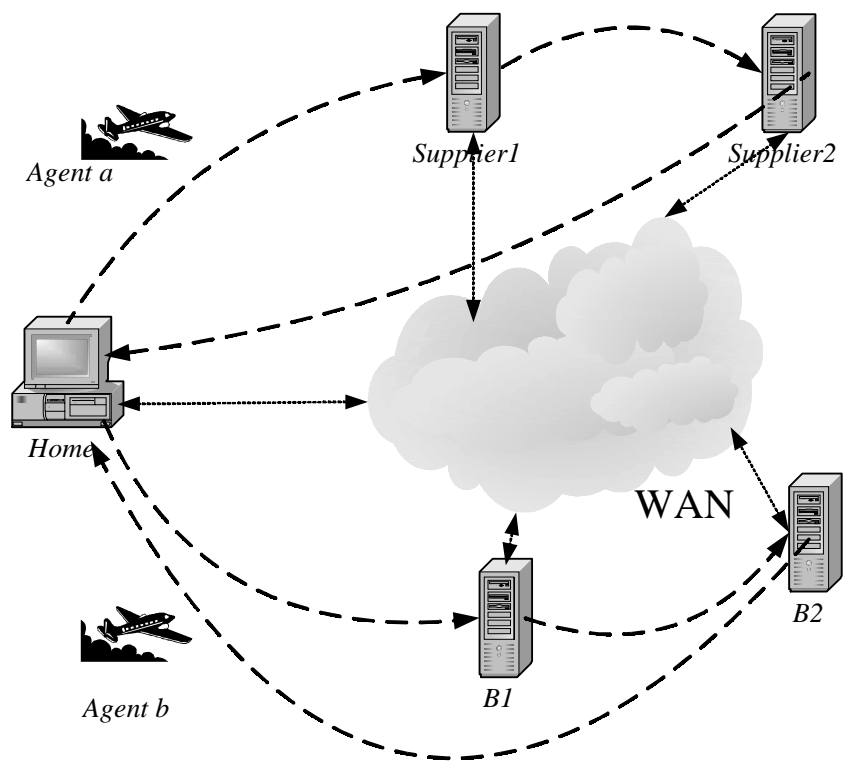

Fig. 11. Experimental environment.

Finally, we tested the detection of the attacks on the itinerary. We carried out the attack where platform $h a_{i}$ sends agent $a$ to a different platform than agent $a$ 's original destination. In the same way, we tested the same attack with an attempt to put the agent on its track. In both cases, the attack was detected during the same step $i$. However, the tests carried out on the other attacks were limited in terms of security features to be verified. Indeed, attacks where there is collaboration between a host on agent $a$ 's itinerary and $a$ host on agent $b$ 's itinerary consist of manipulating the agents. The purpose of this manipulation is to modify the equality result or to alter communication between the agents.

We chose to measure the cost of our protocol on a prototypical mobile agent application - the shopping application described earlier. As seen earlier, the agent departs with a shopping list of three products. It only knows the address of one vendor, called supplierl. The first vendor sends the agent to another vendor since he does not carry one of the products and has trade agreements that allow forwarding the agent to another vendor, called supplier 2 . Agent $a$ has a list of hosts that agent $b$ will visit.

Five machines are used to conduct the experimental study. A Grasshopper platform is installed on each machine. Each platform represents a different site. Two platforms, called Supplierl and Supplier2 constitute agent $a$ 's itinerary. Two other platforms, called $B 1$ and $B 2$ constitute agent $b$ 's itinerary. A fifth platform, called Home, represents the platform of origin. Consequently, agents $a$ and $b$ are created within the Home platform which sends agent $a$ to Supplierl's platform and agent $b$ to B1's platform. Agent $a$ moves from Supplierl to Supplier2 before returning to Home platform. Agent $b$ moves from $B 1$ to $B 2$ before returning to Home platform. Figure 11 illustrates this experimental environment.

Our tests measured both:

- the execution time for each vendor machine visited by the shopping agent;

- the total execution time for the shopping agent and its cooperating agent.

We chose to measure these variables as they reflect some of the advantages of the mobile agent paradigm over the client-server approach. We measured the execution time with the Java method System.currentTimeMillis(). For the first measurement, we call this method upon the arrival of the agent 


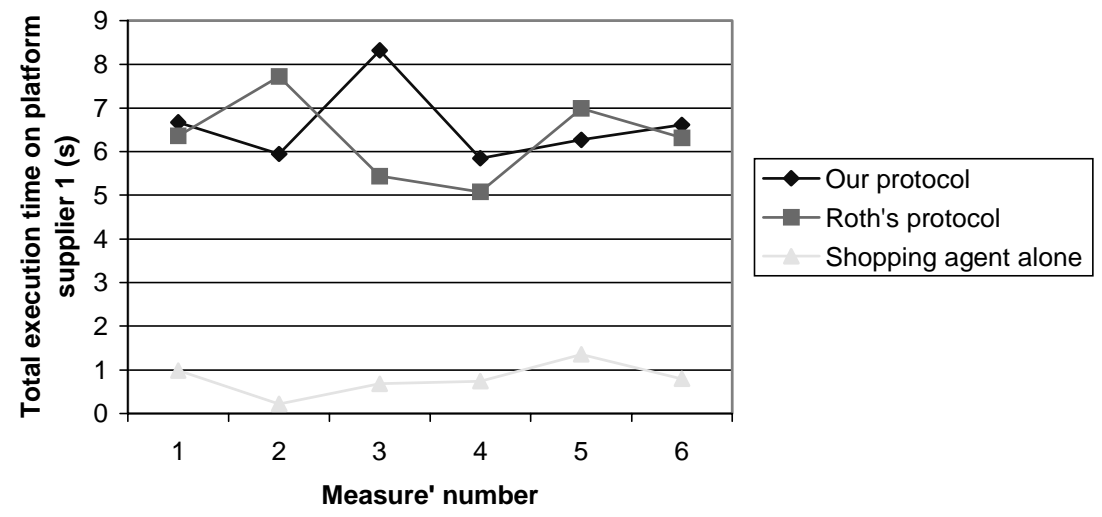

Fig. 12. Execution time on platform Supplierl.

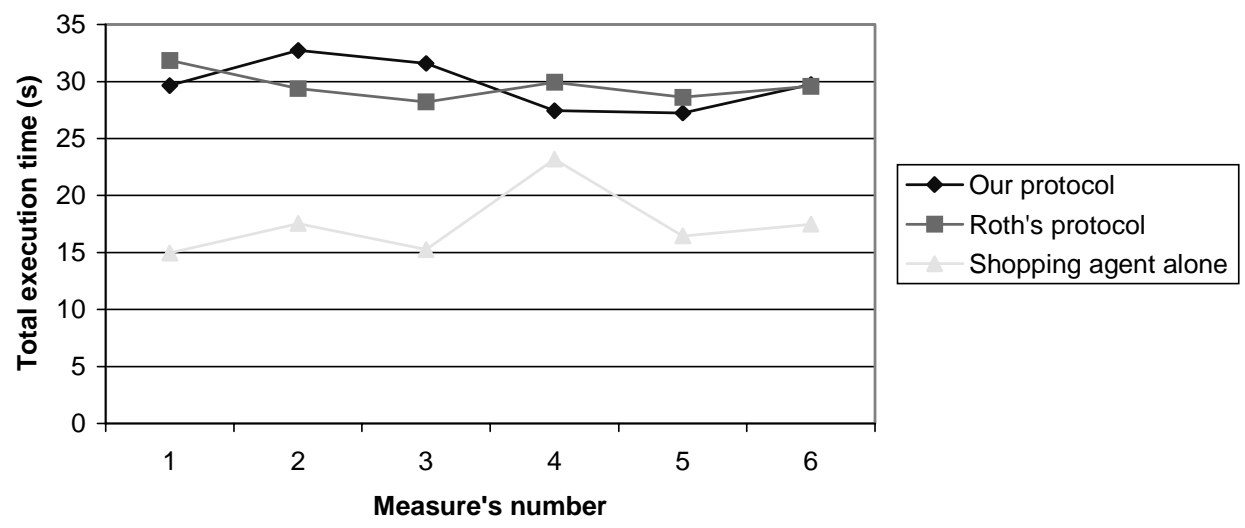

Fig. 13. Total execution time.

on the platform and before it migrates to the next platform. The second measurement corresponds to the difference between the time the agents were created and and the moment where agent $b$ finished the verifications after having returned to its owner's platform. Hence, this measurement corresponds to the total time the task required. We did measurements with three types of agents:

- the shopping agent and its cooperating agent, using our itinerary recording protocol;

- the shopping agent and its cooperating agent, using Roth's protocol;

- only the shopping agent.

Figure 12 shows the execution time for these three situations. It should be noted that there are some important differences in a given category. For example, measurement 3 of our protocol indicates the highest results. We noticed (via Grasshopper) that the thread executing the agent used a minimal priority. The fact that the other threads were executed on the process platform explains the variations in execution time. Moreover, while the execution time differs little between our protocol and Roth's (around $7 \mathrm{~s}$ in both cases), it is much lower for the shopping agent. This is explained by the fact that, in both our protocol and Roth's, the shopping agent establishes an SSL connection with its cooperating agent, while this is not required by the shopping agent alone. However, establishing this connection requires time, since the virtual machine must load many classes in memory and initialize the SSL session. 
Figure 13 shows the total execution time for the three cases. Just as with the execution time on a vendor platform, although our protocol and Roth's have similar values, the shopping agent alone requires less execution time. These results are related to the platform execution time. Note that the total execution time reflects both the execution time on each platform and the total migration time. Since the network's features do not change, the migration time would vary little from one measurement to another. Similarly, the difference among the migration times of three agents is small, since the agents are of similar sizes in relation to the network flow; moreover, in all three cases, we use an SSL connection to transport the agents.

We also find a large difference between the relative gap of our protocol and the shopping agent, for the execution on the vendor's platform (700\%) and the total execution time (70\%). As mentioned above, this is due to the SSL connection which requires much more time.

\section{Conclusion}

This paper presented a mobile agent itinerary recording protocol. This protocol gives a secure method of recording the identities of the platforms on which a mobile agent is executed. This problem is difficult to solve with a single agent since the data transmitted are prone to manipulation by malicious platforms, which is the reason why Roth [15] introduced the concept of cooperating agents.

We have designed an itinerary recording protocol using the concept of cooperating agents and described in details the behavior of each of the two cooperating agents, as well as the procedures selected to establish the identities of the offenders when an attack is detected, as already done by Roth. To show the security properties of our protocol, we reviewed a set of potential attacks. For each attack, we described the moment of detection and, when possible, we designated the offenders. Thus, we have been able to verify that our protocol detects the attack in cases where there is collaboration between a platform on the cooperating agents' itinerary and another on the agent's itinerary. This type of attack is outside the scope of those addressed by Roth.

Finally, we implemented our protocol and Roth's on a mobile agent platform. To test these implementations, we chose a mobile agent shopping application with a random itinerary. This is a classical application using the mobile agent paradigm where itinerary recording is critical. We tested our protocol on a set of possible attacks and the results confirm our assumption in terms of offender designation and moment of detection. We compared three implementations (the shopping agent with our protocol, with Roth's protocol, and the shopping agent alone) and measured the execution time of the shopping agent on each platform and the total execution time. Results show that our protocol costs slightly more than Roth's in terms of execution time; however, our protocol detects more attacks than Roth's. The use of an itinerary recording protocol (either ours or Roth's) adds a significant cost in terms of execution time as well as in terms of network load. Thus, our protocol is not suitable in cases where cost is critical.

Future research could strive to create an algorithm that would allow unmasking the offender of a kidnapped mobile agent: the case in which the cooperating agent's timer expires. With our protocol, we know that the kidnapping offender is either the platform from which the agent communicates before the latter could migrate, or the platform where the agent was meant to be sent the last time it communicated with the cooperating agent.

\section{References}

[1] A.O. Freier, P. Karlton and P. Kocher, The SSL Protocol, Version 3.0, Internet Draft, March, 1996, http://home.netscape. com/eng/ssl3/ssl-toc.html. 
[2] C. Meadows, Detecting Attacks on Mobile Agents, DARPA Workshop on Foundations for Secure Mobile Code Workshop, March, 1997, http://www.cs.nps.navy.mil/research/languages/statements/meadows.ps

[3] D. Chess, Security Issues in Mobile Code, in: Mobile Agents and Security, G. Vigna, ed., Springer-Verlag, Berlin, Germany, 1998, pp. 1-15.

[4] D. Westhoff, M. Schneider, C. Unger and F. Kaderali, in: Protecting a Mobile Agent's Route Against Collusions, Heys and Adams, eds, SAC'99, Springer-Verlag, LNCS, 1758, pp. 215-225.

[5] F.B. Schneider, Towards Fault-Tolerant and Secure Agentry, in Proceedings of the 11th International Workshop on Distributed Algorithms, Germany, September, 1997, pp. 1-14.

[6] F. Hohl Time Limited Blackbox Security: Protecting Mobile Agents from Malicious Hosts, in: Mobile Agents and Security, G. Vigna, ed., Springer-Verlag, Berlin, Germany, 1998, pp. 92-113.

[7] F. Hohl, A Framework to Protect Mobile Agents by Using References States, in Proceedings of ICDCS 2000, 2000, ftp://ftp.informatik.uni-stuttgart.de/pub/library/ncstrl.ustuttgart fi/TR-2000-03/TR-2000-03.ps.gz.

[8] G. Cugola, C. Ghezzi, G. Picco and G. Vigna, Analyzing Mobile Code Languages, in Mobile Objet Systems: Towards the Programmable Internet, Springer-Verlag, Berlin, Germany, 1997, 93-111.

[9] G.C. Necula and P. Lee, Safe, Untrusted Agents Using Proof-Carrying Code, in: Mobile Agents and Security, G. Vigna, ed., Springer-Verlag, Berlin, Germany, 1998, pp. 61-91.

[10] Institute of Applied Information Processing and Communications, Javadoc for IAIK-JCE 3.01, Online Documentation, 2002, http://jce.iaik.tugraz.at/products/01 jce/documentation/index.php.

[11] M.S. Greenberg, J.C. Byington, T. Holding and D.G. Harper, Mobile Agents and Security, IEEE Communications Magazine (July, 1998), 76-85.

[12] R. Oppliger, Security Issues related to mobile code and agent-based systems, Computer Communications 22 (1999), $1165-1170$.

[13] T. Sander and C.F. Tschudin, Protecting Mobile Agents Against Malicious Host, in: Mobile Agents and Security, G. Vigna, ed., Springer-Verlag, Berlin, Germany 1998, pp. 44-60.

[14] V. Pham and A. Karmouch, Mobile Software Agents: An Overview, IEEE Communications Magazine (July, 1998), 26-37.

[15] V. Roth, Mutual Protection of Co-operating Agents, in: Secure Internet Programming, Vitek and Jensen, eds, SpringerVerlag, Berlin, Germany, 1998, pp. 26-37.

[16] V. Roth, On the Robustness of some Cryptographic Protocols for Mobile agent Protection, in Proceedings of the 5th International Conference on Mobile Agent, MA 2001, Atlanta, GA, USA, December 2001, Springer-Verlag, 1-14.

[17] V. Roth, Empowering Mobile Software Agents, in Proceedings of the 6th IEEE Mobile Agents Conference, MA 2003, Springer-Verlag, October, 2002, 47-63.

Guillaume Allée received the B. Eng. degree in Computer Engineering at École Supérieure d'électricité (Supélec), Paris, and a master degree in Computer Engineering at Ecole Polytechnique de Montréal. His research interest include mobile computing and networking.

Samuel Pierre received the B. Eng. degree in civil engineering in 1981 from École Polytechnique de Montréal, Québec, the B. Sc. and M.Sc. degrees in mathematics and computer science in 1984 and 1985, respectively, from the UQAM, Montréal, the M.Sc. degree in economics in 1987 from the Université de Montréal, and the Ph.D. degree in Electrical Engineering in 1991 from École Polytechnique de Montréal. Dr. Pierre is currently a Professor of Computer Engineering at École Polytechnique de Montréal where he is Director of the Mobile Computing and Networking Research Laboratory (LARIM) and NSERC/Ericsson Industrial Research Chair in Next-generation Mobile Networking Systems. He is the author of four books, co-author of two books and seven book chapters, as well as over 250 other technical publications including journal and proceedings papers. He received the Best Paper Award of the Ninth International Workshop in Expert Systems \& their Applications (France, 1989), a Distinguished Paper Award from OPNETWORK'2003 (Washington, USA). One of these coauthored books, Télécommunications et Transmission de données (Eyrolles, 1992), received special mention from Telecoms Magazine (France, 1994). His research interests include wireline and wireless networks, mobile computing, performance evaluation, artificial intelligence, and electronic learning. He is a Fellow of Engineering Institute of Canada, senior member of IEEE, a member of ACM and IEEE Communications Society. He is an Associate Editor of IEEE Communications Letters and IEEE Canadian Review, and he serves on the editorial board of Telematics and Informatics published by Elsevier Science.

Roch H. Glitho [SM] (http://www.ece.concordia.ca/ glitho/) received a Ph.D. (Tekn. Dr.) in tele-informatics (Royal Institute of Technology, Stockholm, Sweden) and M.Sc. degrees in business economics (University of Grenoble, France), pure mathematics (University Geneva, Switzerland), and computer science (University of Geneva). He works in Montreal, Canada, as an expert in service engineering at Ericsson, and as an adjunct associate professor at Concordia University. In the past he worked as a senior specialist in network management for Ericsson Telecom in Stockholm, and as an R\&D engineer for a computer manufacturer 
in Oslo, Norway. His industrial experience includes research, international standards setting (e.g. contributions to ITU-T, ETSI, TMF, ANSI, TIA, and 3GPP), product management, project management, systems engineering and software/firmware design. He is the Editor-in-Chief of IEEE Communications Magazine and serves as a Technical editor for the Journal of Network and Systems Management (JNSM) published by Plenum/Kluwer. He is also an IEEE distinguished lecturer. In the past (1998-2000), he served as the Editor-In-Chief for the IEEE Communications Surveys \& Tutorials on-line magazine. His research areas include service engineering, network management, signaling and mobile code. In these areas, he has authored more than 30 peer-reviewed papers, more a dozen of which have been published in well-known refereed journals. He has also guest-edited some 10 special issues of refereed journals and has more than 20 patents in the aforementioned areas.

Abdelmorhit EL RHAZI received a bachelor degree in computer engineering from Ecole Mohammadia d' Ingenieurs (Morocco) in 1995, an M.Sc. degree in computer engineering in 2003 from Ecole Polytechnique of Montreal. He worked as an computer engineer for seven years. He is currently completing his Ph.D. at Ecole Polytechnique of Montreal. His research interests include security of computer systems, mobile agents and Sensor Networks. 

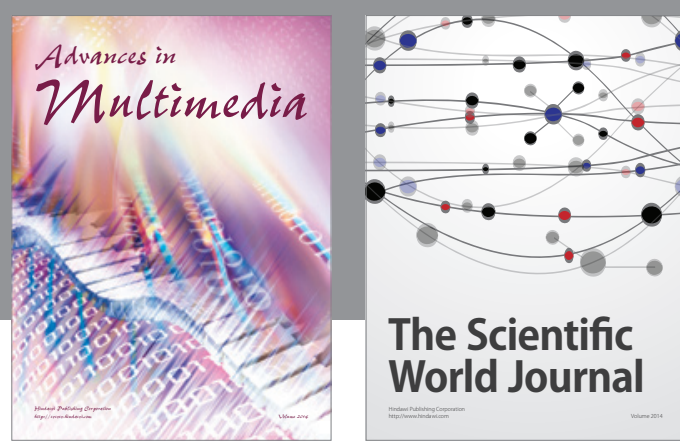

The Scientific World Journal
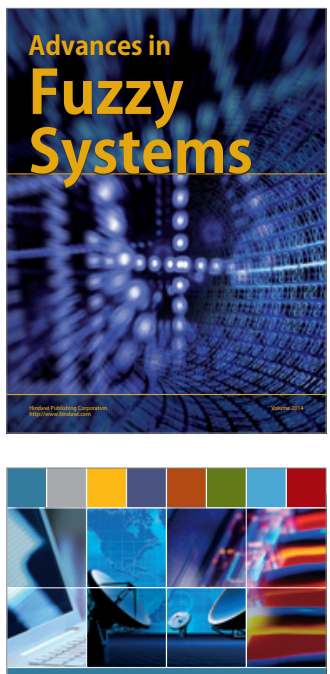

Computer Networks and Communications
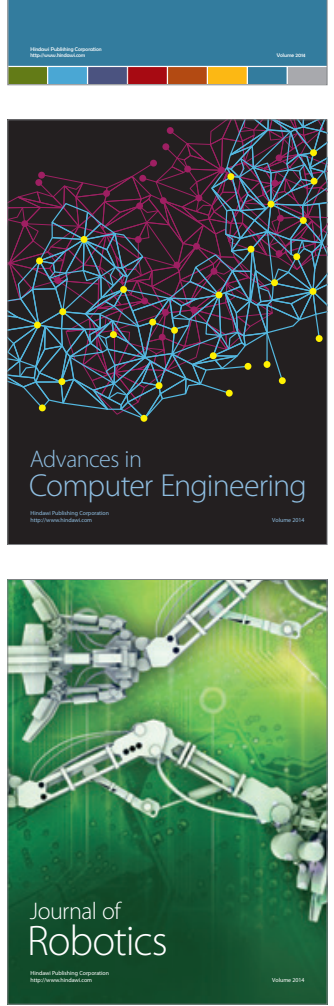
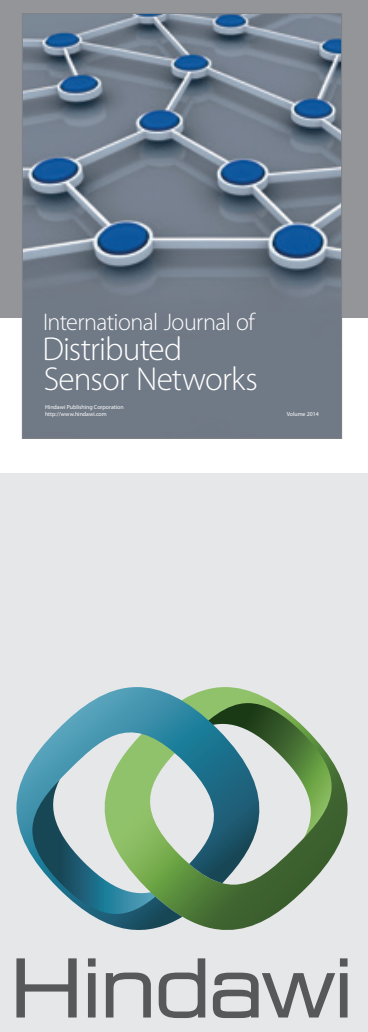

Submit your manuscripts at

http://www.hindawi.com
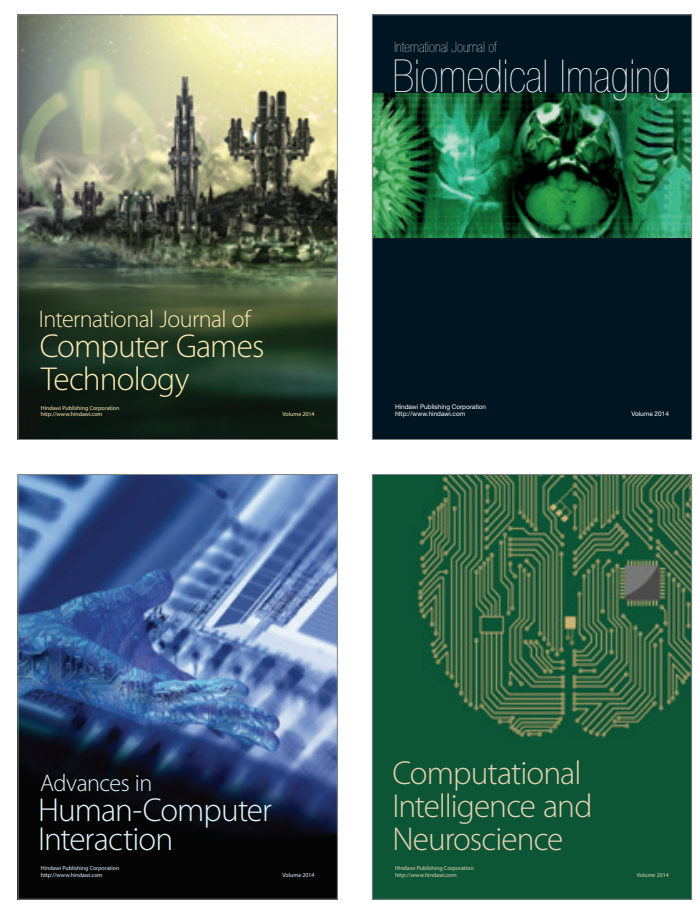
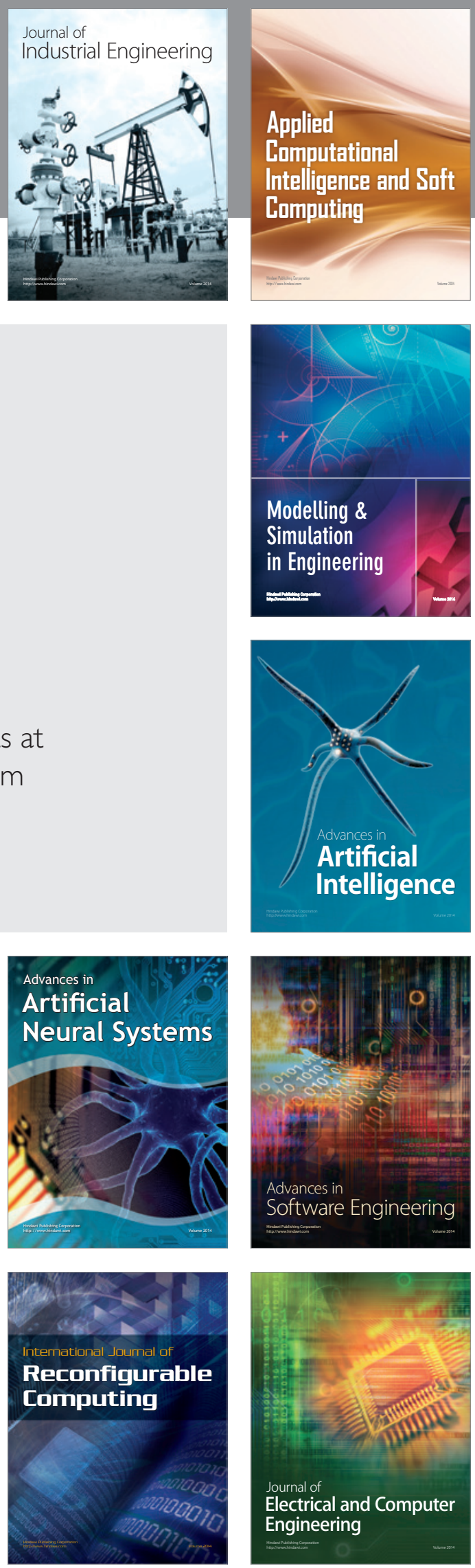